Research Paper

\title{
Mutational Characterization of Colorectal Cancer from Korean Patients with Targeted Sequencing
}

\author{
Jongmin Lee, MS1,2*; Sangtae Choi, MD, PhD ${ }^{*}$; Donghae Jung, MD, PhD ${ }^{4}$; YunJae Jung, MD, PhD 2,5; Jung \\ Ho Kim, MD, PhD' ${ }^{6}$; Sungwon Jung, PhD ${ }^{1,2,7}$; Won-Suk Lee, MD, PhD ${ }^{3 凶}$ \\ 1. Gachon Institute of Genome Medicine and Science, Gachon University Gil Medical Center, Incheon, Republic of Korea \\ 2. Gachon Advanced Institute for Health Science and Technology, Gachon University, Incheon, Republic of Korea \\ 3. Department of Surgery, Gachon University College of Medicine Gil Medical Center, Incheon, Republic of Korea \\ 4. Department of Pathology, Gachon University College of Medicine Gil Medical Center, Incheon, Republic of Korea \\ 5. Department of Microbiology, Gachon University College of Medicine, Incheon, Republic of Korea \\ 6. Department of Internal Medicine, Gachon University College of Medicine Gil Medical Center, Incheon, Republic of Korea \\ 7. Department of Genome Medicine and Science, Gachon University College of Medicine, Incheon, Republic of Korea \\ * These authors equally contributed as first authors. \\ $\triangle$ Corresponding authors: Sungwon Jung (phone: +82-32-458-2740; e-mail: sjung@gachon.ac.kr) and Won-Suk Lee (phone: +82-32-458-3272; e-mail: \\ lws@gilhospital.com)
}

(1) The author(s). This is an open access article distributed under the terms of the Creative Commons Attribution License (https://creativecommons.org/licenses/by/4.0/). See http://ivyspring.com/terms for full terms and conditions.

Received: 2021.04.06; Accepted: 2021.10.09; Published: 2021.10.28

\begin{abstract}
Purpose: Effective treatment of colorectal cancer could benefit from understanding molecular characteristics including mutation profiles of important genes. This study aimed to explore the molecular characteristics of colorectal cancer based on next generation sequencing.

Methods: The mutational characteristics by targeted next generation sequencing in 172 colorectal tumor samples from Korean patients were evaluated to explore their associations with clinical features. Targeted sequencing of 375 genes was performed with an average target-depth of $800 \mathrm{X}$.

Results: TP53 and APC showed higher mutation frequencies from the left-sided tumors, while CTNNB1 were more frequent from the right-sided tumors. The tumor suppressor NOTCHI and the DNA strand break repair gene PALB2 were more frequently mutated in early onset tumors. KRAS and PTEN mutations were more frequent from patients with advanced cancers by cancer antigen markers. TP53 and BRAF mutations were more frequent from patients of T3 and T4 stages, where their variant allele fractions were generally higher in T4 tumors, implying that advanced tumors have higher fraction of cancer cells with TP53 and BRAF mutations. Mutational profiles of these patients were also assessed with other clinical features. Comparison of mutational characteristics with the Caucasian subjects from independent data showed that the identified mutational characteristics are largely Korean-specific except for a few key colorectal cancer genes.

Conclusion: Next generation sequencing-based targeted sequencing can provide valuable information on molecular characterization of colorectal cancer patients, and its clinically relevant information can provide benefits to better understand colorectal cancer.
\end{abstract}

Key words: Colorectal cancer, targeted sequencing, mutation, Korean population

\section{Introduction}

The clinical impact of tumor location (i.e left VS. right sidedness) on patient survival and response to chemotherapies has been shown in large clinical trials [1]. However, the underlying molecular biology explaining such differences has not been clearly defined. The anatomical difference can be considered from embryological origin. The right colon receives blood supply from superior mesenteric vessels, a midgut structures from the mid-duodenum to the mid-transverse colon, whereas the inferior mesenteric artery supplies hindgut structures from the mid-transverse colon to the rectum. Aside from this 
embryological point of difference, colon cancer has been recently subcategorized to the following CMS subtypes: 1) microsatellite instability immune $(14 \%)$, 2) canonical (37\%), 3) metabolic (13\%), 4) mesenchyme (23\%) and 5) mixed features (13\%) [2]. The differential distribution of the five classes in various anatomic regions suggests biological differences in the right-sided colon and left-sided colon cancer. It has been shown that right-sided colon cancer patients were older, had larger tumor sizes and more advanced tumor stages, were more often female, and had poorly differentiated tumors [1]. Petrelli et al. [3] revealed in their large cohort study that left-sided colon cancer was associated with a significantly reduced risk of death, which was independent of stage, race, and adjuvant chemotherapy. However, questions still remain despite such huge clinical differences, mainly because molecular understanding is as yet scattered and not comprehensive. In this study, we investigated the genetic aberrations in clinical colon cancer samples to further delineate these molecular differences.

Multiple studies investigated the association between mutation frequencies and clinical characteristics including tumor locations from colorectal cancer. Lee et al. [4] compared the frequencies of somatic mutations between left-sided and right-sided tumors. Lieu et al. [5] investigated the differences in gene mutations with varying ages of patients. Shen et al. [6] evaluated the prognostic impact of different mutation profiles in stage II and III colorectal cancer patients. Poulos et al. [7] compared the mutation profiles across colorectal cancer patients with varying MSI status. Bordonaro and Lazarova [8] investigated the mutation profiles with varying obesity status of patients, and suggested association between obesity and lower mutation threshold. However, there has been limited study on the mutational and clinical characteristics of colorectal cancer from East Asia including Korean populations, while Byun et al. [9] assessed the mutation status of KRAS based on the location of primary tumors from 1,115 Korean patients. Therefore, there are further unmet needs to investigate the molecular characteristics along with accompanying clinical information for East Asian populations as well as for region or nation-specific populations. In this study, tumor samples from 172 Korean patients with colorectal cancer were examined with deep targeted sequencing to evaluate whether their various clinical characteristics are related with different molecular characteristics. We assessed the single-nucleotide variations from tumor samples to select potentially pathogenic somatic mutations. The mutational characteristics of the 172 patients were evaluated in association with multiple clinical features, including tumor locations, ages, cancer antigen marker levels, and the depth of tumor invasion. The identified mutational characteristics were also compared with that of the Caucasian colorectal cancer patients from independent data to show its ethnic specificity. The identified associations between somatic mutations and clinical features of Korean colorectal cancer patients from this study can provide beneficial information to better understand the molecular characteristics of colorectal cancer.

\section{Materials and Methods}

\section{Patients and clinical data collection}

FFPE samples of primary tumor tissues were obtained from 172 colorectal cancer patients and prepared for mutation profiling with deep targeted sequencing. The tumor tissue samples were retrieved from patients at Gachon University Gil Medical Center (Incheon, Republic of Korea) between April 2017 and April 2019. Patients were enrolled only when colorectal cancer was detected by abdominal and pelvic computed tomography to acquire enough specimens for this study. The detailed demographic and clinical characteristics of the 172 patients are given in Table 1 . The experiments conducted on patient samples were approved by the institutional review board of Gachon University Gil Medical Center (IRB No. GCIRB2019-223, A study to identify the relationship between genetic variation of colon cancer and clinicopathological characteristics). All experiments and analysis procedures were performed in accordance with the relevant guidelines and regulations. Written informed consents were obtained from all participating patients.

Regarding the laboratory measurement of CA 19-9 and CEA levels, peripheral blood samples were obtained from participating patients at an average $8 \pm$ 2 days prior to the operation. All the assays were performed in a single laboratory by following manufacturers' guidelines, where the RIAMAT SR-300 kit (Stratec, Germany) was used for the serum CEA levels, and the ADVIA Centaur CA 19-9 assay (Siemens Medical Solutions Diagnostics, US) was used to measure CA 19-9 levels. For the classification of MSI status, a panel of microsatellite markers, including the mononucleotide repeat markers BAT25 and BAT26, and the dinucleotide repeat markers D5S346, D2S123, and D17S250, were used according to the international guidelines. Instability in more than $40 \%$ of the analyzed markers was defined as MSI-high and classified as MSI in this study. Tumors with instability in less than $40 \%$ of the markers 
(MSI-low) and tumors without instability were classified as MSI [10].

Table 1. Demographic and clinical landscape of the 172 CRC patients in this study

\begin{tabular}{|c|c|c|}
\hline & Characteristics & Number of patients (\%) \\
\hline \multirow[t]{2}{*}{ Sex } & Male & $112(65.1 \%)$ \\
\hline & Female & $60(34.9 \%)$ \\
\hline \multirow[t]{2}{*}{ Tumor sidedness } & Left & $125(72.7 \%)$ \\
\hline & Right & $47(27.3 \%)$ \\
\hline \multirow[t]{3}{*}{ Age } & $>=50$ & $157(91.3 \%)$ \\
\hline & $<50,>=45$ & $11(6.4 \%)$ \\
\hline & $>45$ & $4(2.3 \%)$ \\
\hline \multirow[t]{2}{*}{ CA19-9 } & $>=25$ & $35(20.3 \%)$ \\
\hline & $<25$ & $137(79.7 \%)$ \\
\hline \multirow[t]{2}{*}{ CEA } & $>=5$ & $53(30.8 \%)$ \\
\hline & $<5$ & $119(69.2 \%)$ \\
\hline \multirow[t]{4}{*}{ TNM T Stage } & $\mathrm{T} 1$ & $19(11.0 \%)$ \\
\hline & $\mathrm{T} 2$ & $27(15.7 \%)$ \\
\hline & $\mathrm{T} 3$ & $94(54.7 \%)$ \\
\hline & $\mathrm{T} 4$ & $32(18.6 \%)$ \\
\hline \multirow[t]{2}{*}{ TNM N Stage } & No & $93(54.1 \%)$ \\
\hline & N1 & $79(45.9 \%)$ \\
\hline \multirow[t]{2}{*}{ TNM M stage } & M0 & $144(83.7 \%)$ \\
\hline & M1 & $28(16.3 \%)$ \\
\hline \multirow[t]{2}{*}{ MSI } & MSS & $165(95.9 \%)$ \\
\hline & MSI & $7(4.1 \%)$ \\
\hline \multirow{2}{*}{$\begin{array}{l}\text { Lymphovascular } \\
\text { invasion (LVI) }\end{array}$} & Without LVI & $81(47.1 \%)$ \\
\hline & With LVI & $91(52.9 \%)$ \\
\hline \multirow[t]{2}{*}{ Diabetes mellitus } & Without diabetes mellitus & $134(77.9 \%)$ \\
\hline & With diabetes mellitus & $38(22.1 \%)$ \\
\hline \multirow[t]{2}{*}{ BMI } & $>=25$ & $45(26.2 \%)$ \\
\hline & $<25$ & $127(73.8 \%)$ \\
\hline \multirow{3}{*}{$\begin{array}{l}\text { ECOG performance } \\
\text { status }\end{array}$} & 0 & $51(29.7 \%)$ \\
\hline & 1 & $97(56.4 \%)$ \\
\hline & 2 & $24(13.9 \%)$ \\
\hline \multirow{2}{*}{$\begin{array}{l}\text { Synchronous lung } \\
\text { metastasis }\end{array}$} & No & $169(98.3 \%)$ \\
\hline & Yes & $3(1.7 \%)$ \\
\hline \multirow{2}{*}{$\begin{array}{l}\text { Synchronous liver } \\
\text { metastasis }\end{array}$} & No & $155(90.1 \%)$ \\
\hline & Yes & $17(9.9 \%)$ \\
\hline \multirow[t]{2}{*}{ Bowel obstruction } & No & $128(74.4 \%)$ \\
\hline & Yes & $44(25.6 \%)$ \\
\hline
\end{tabular}

\section{Molecular characterization with targeted sequencing}

The molecular characterization of tumors was performed by identifying genomic mutations with targeted sequencing. Sequencing was performed on genomic DNA extracted from FFPE tumor samples using the NextSeq platform (Illumina, Inc., San Diego, CA), and no matched normal tissue was sequenced. The sequencing was performed at LabGenomics (Seongnam, Republic of Korea) using the
CancerSCAN level 2 targeted panel. For sequencing, specific regions of the genome spanning $2.599 \mathrm{Mb}$ were amplified to cover 375 whole-gene targets (Supplementary Table S1). The targeted regions were sequenced with average target-depth of $800 \mathrm{X}$, and more than $94 \%$ of sequenced bases showed quality scores higher than Q30.

It is known that mutational profiling with FFPE samples can include false-positive mutations, where base changes of $C$ to $T$ or $G$ to $A$ can occur with low frequency due to cytosine deamination in the process of manufacturing FFPE samples [11]. In order to exclude such potentially false-positive mutations due to the process of manufacturing FFPE samples, mutations of base changes $\mathrm{C}$ to $\mathrm{T}$ and $\mathrm{G}$ to $\mathrm{A}$ with variant allele fraction less than $5 \%$ were excluded from the downstream analysis [12].

\section{Filtering potential somatic mutations}

In order to consider only potentially somatic mutations that affect protein formation and functions among the identified SNVs and short insertions/deletions, genetic variants whose positions are other than exons or splicing sites as well as synonymous variants were discarded from further analysis. Among the remaining variants, variants with minor allele frequencies greater than 0.01 from multiple variant databases (ESP6500, ExAC and 1000 genome project) were further discarded. As a final measure to further eliminate potentially false positive somatic mutations, the mutation frequencies of individual genes from the variants were computed from the 172 patients in this study, and they were compared with the mutation frequencies from the COSMIC database for colorectal cancer. If a gene showed more than 3-fold difference in mutation frequencies between our data and the COSMIC database or its statistical significance p-value was less than 0.05 (by Fisher's exact test), it was considered as a false-positive finding and discarded from the results.

\section{Statistical methods}

Fisher's exact test was applied to evaluate the statistical significance of difference in mutation frequencies between two patient groups, and genes showing p-value $<0.05$ were declared to show differences. TML of each sample was measured with the number of nonsynonymous mutations per $1 \mathrm{Mb}$ sequenced bases, where expected hotspot mutations [13] were discarded before computation to avoid potential bias of enriched mutations from using the targeted sequencing panel. Welch's t-test was used to test if left- and right-sided tumors show differences in TML.

In order to evaluate if the identified mutational 
characteristics for each clinical variable from our study is specific to Korean populations, we compared our results with the mutational characteristics of the Caucasian population from the TCGA colorectal cancer data [14]. Among the clinical variables that we investigated, information on tumor location, subject ages, BMI, TNM stages were also available from TCGA. Somatic mutation information for the 207 Caucasian subjects from the TCGA colorectal cancer data was used to identify the clinical variable-associated mutational characteristics. For each clinical variable, Caucasian subjects were grouped based on the same criteria with our Korean subjects, and Fisher's exact test was performed on mutation frequencies between subject groups. The same p-value threshold of 0.05 was used to declare statistical significance on the difference of mutation frequencies for the Caucasian subjects.

\section{Results}

\section{Discrepancy in genomic characteristics of Korean colorectal cancers by tumor locations}

We compared the mutation frequencies between 125 patients with left-sided tumors and 47 patients with right-sided tumors. When comparing the mutation frequencies of selected genes (BRAF, KRAS, TP53, APC, PIK3CA, CTNNB1, ATM, PTEN and BRCA1) that are commonly known for colorectal cancer [15] (Fig. 1A), TP53 (left $=80 \%$, right $=55.3 \%$; p $=0.002$ ) and APC (left $=77.6 \%$, right $=57.5 \%$; $=$ 0.013) showed significant difference between left-sided and right-sided tumors with higher mutation frequencies on the left-sided tumors. On the contrary, CTNNB1 (left $=3.2 \%$, right $=12.8 \%$; $p=$ 0.026 ) showed higher mutation frequency on the right-sided tumors than the left-sided tumors. Other study also investigated the differences in mutation frequencies of colorectal cancer based on tumor location [15]. In that study, TP53 also showed significantly higher mutation frequency on the left-sided tumors than the right-sided tumors, which is consistent with our result. Their results also showed the same left/right-side preference of mutation frequencies for APC and CTNNB1, but their result did not accompany statistical significance. This slight difference between two studies may be due to the heterogeneity in genomic characteristics between subjects from Korean populations of our study and the subjects from the previous study. We also investigated other genes that showed large differences in mutation frequencies between the left-sided and right-sided tumors (Fig. 1B). FBXW7 (left $=20 \%$, right $=6.4 \% ; p=0.036$ ), which is known as a tumor suppressor gene, showed a significantly higher mutation frequency from the left-sided tumors than the right-sided tumors. PRKDC, ATRX, and NSD1 showed higher mutation frequencies from the right-sided tumors than the left-sided tumors. PRKDC (left $=15.2 \%$, right $=34 \% ; p=0.01)$ is a protein kinase and plays a role in DNA double strand break repair [16]. ATRX (left $=3.2 \%$, right $=17 \%$; $\mathrm{p}=0.004$ ) mutations have been reported in various types of cancer, and it is known to be associated with MSI [17]. These higher mutation frequencies of PRKDC and ATRX from the right-sided tumors than the left-sided may be related to the more frequent observation of MSI-high cases from the colorectal cancer patients with right-sided tumors. NSD1 (left $=4.8 \%$, right $=$ $17 \% ; \mathrm{p}=0.023$ ) is known to play a role in histone methylation and its inactivation has been reported to lead to multiple cancers [18].

TML of each sample was calculated, and its distribution was compared between the left-sided and right-sided tumors (Fig. 1C). Difference between the two TML distributions does not show statistical significance ( $p=0.059$ by Welch's T-test), but the right-sided tumors including samples of very high mutational burdens show higher mutational burdens than the left-sided tumors on average (TML of right-sided tumors $=7.41$ nonsynonymous mutations $/$ megabase, TML of left-sided tumors $=4.98$ nonsynonymous mutations/megabase). This may be also related to frequent MSI-High observations from right-sided colorectal cancers.

\section{Frequent mutations in early onset colorectal cancer}

To identify mutations associated with early-onset colorectal cancer, we compared the mutation frequencies of 15 patients with ages under 50 (U50) and 157 patients with ages 50 or older (O50) (Fig. 2A). NOTCH1(U50 $=40 \%$, O50 $=9.6 \% ; \mathrm{p}=$ $0.004), \operatorname{RB} 1(\mathrm{U} 50=20 \%, \mathrm{O} 50=1.3 \% ; \mathrm{p}=0.045)$, ASXL1 $(\mathrm{U} 50=33.4 \%, \mathrm{O} 50=5.7 \% ; \mathrm{p}=0.003)$ and IGF2R(U50 $=26.7 \%$, O50 $=8.3 \% ; \mathrm{p}=0.045)$, which have been reported to be associated with various cancers as tumor suppressors [19-22], showed high frequencies of mutations in younger patients (U50). FANCI (U50 $=33.4 \%$, O50 $=8.3 \% ; \mathrm{p}=0.011)$ and PALB2 (U50 $=26.7 \%$, O50 $=5.1 \% ; p=0.012)$, where they are known to play roles in repairing DNA double strand breaks [23, 24], also showed high frequencies of mutations from the younger U50 group. With this observation of higher mutation frequencies of some tumor suppressors and DNA repair genes, we further assessed the frequencies of mutations with more stringent categorization with a lower threshold of age 40 (four patients with ages under 40 (U40) VS. 168 patients with ages of 40 or older (O40)) (Fig. 2B). From 
the comparison, there were significant differences in mutation frequencies of NOTCH1 $(\mathrm{U} 40=75 \%, \mathrm{O} 40=$ $10.7 \% ; \mathrm{p}=0.006)$ and PALB2 (U40 $=50 \%, \mathrm{O} 40=6 \%$; $\mathrm{p}$ $=0.024)$. As these tumor suppressor and repairer of DNA strand break show higher mutation frequencies

\section{A}
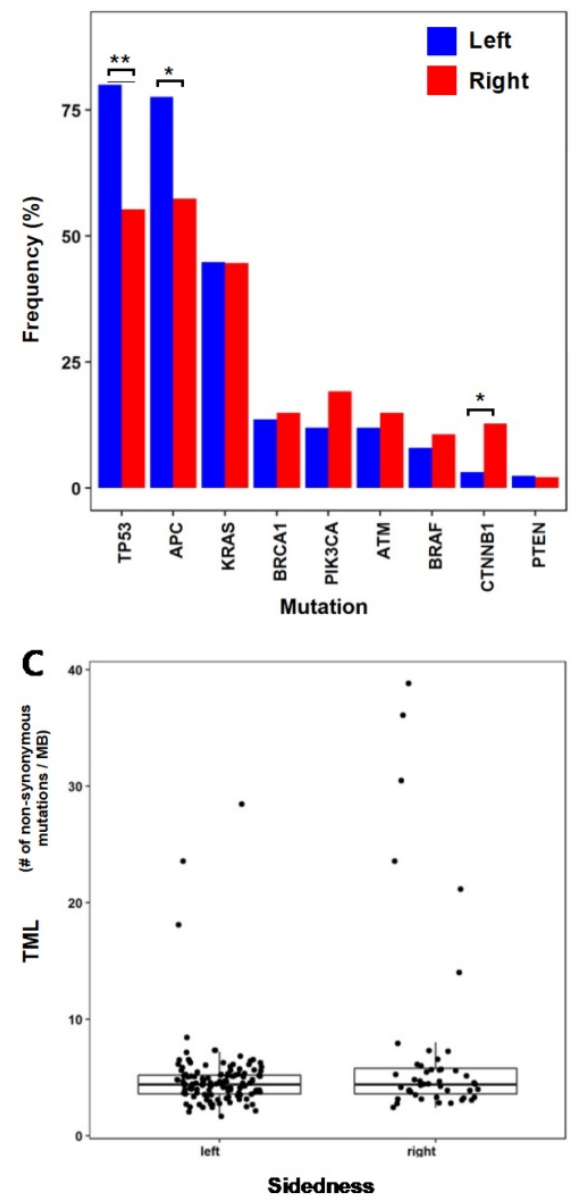

in younger patients, they may be involved in early initiation and development of colorectal cancer by losing tumor suppressing function and allowing more DNA structure alterations.

B

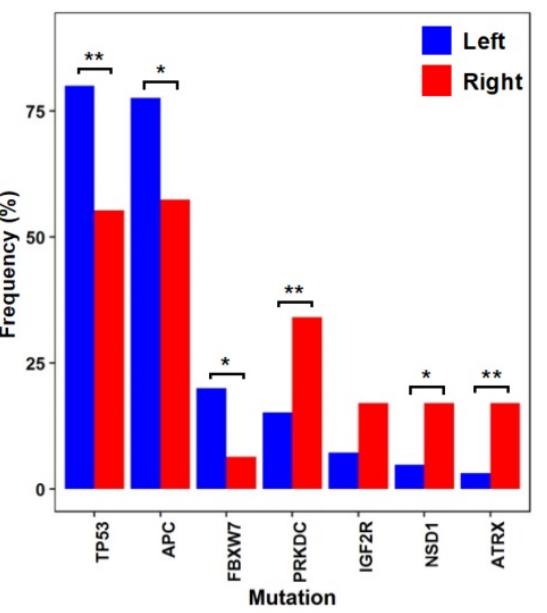

Figure 1. Differences in mutational characteristics based on tumor locations $(*: p<0.05, * *: p<0.01)(A)$ Mutation frequencies of common colorectal cancer genes for the left-sided and right-sided tumors (B) Mutation frequencies of selected genes with large frequency differences (C) Tumor mutational load (TML) of the left-sided and the right-sided tumors
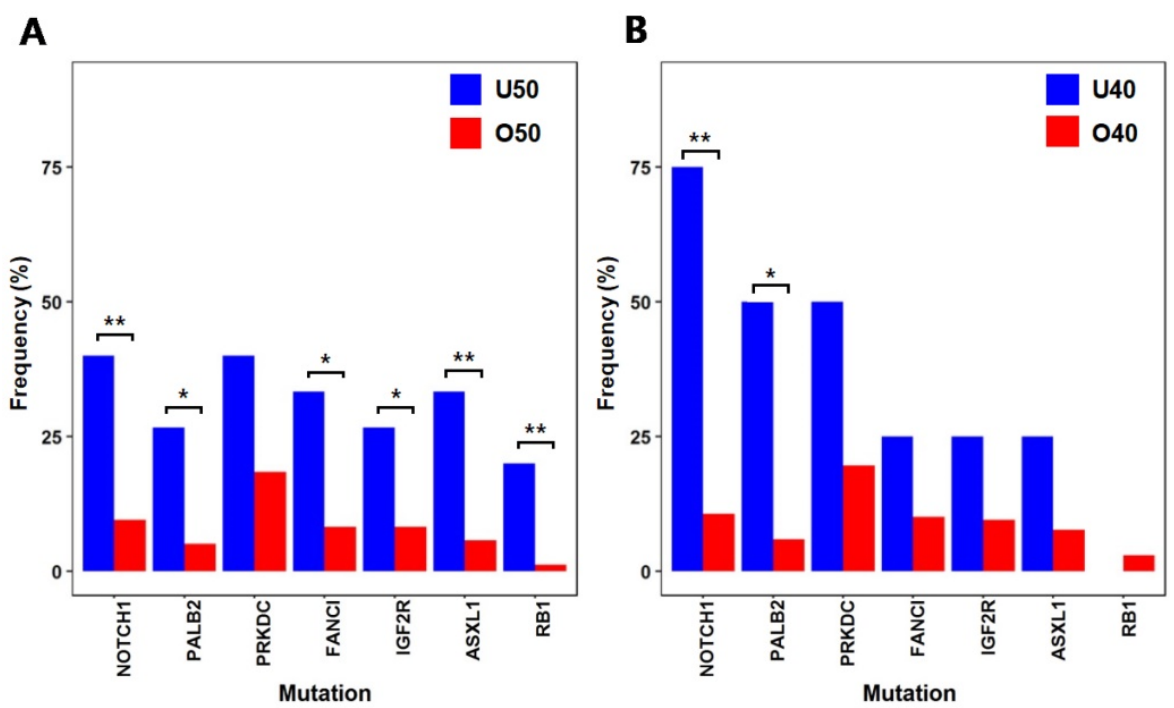

Figure 2. Selected genes that show large differences in mutation frequencies based on patients' ages $\left({ }^{*}: \mathrm{p}<0.05\right.$, ** $\left.: \mathrm{p}<0.01\right)(\mathrm{A})$ Mutation frequencies from two patient groups classified by age 50 (B) Mutation frequencies from two patient groups classified by age 40 
A

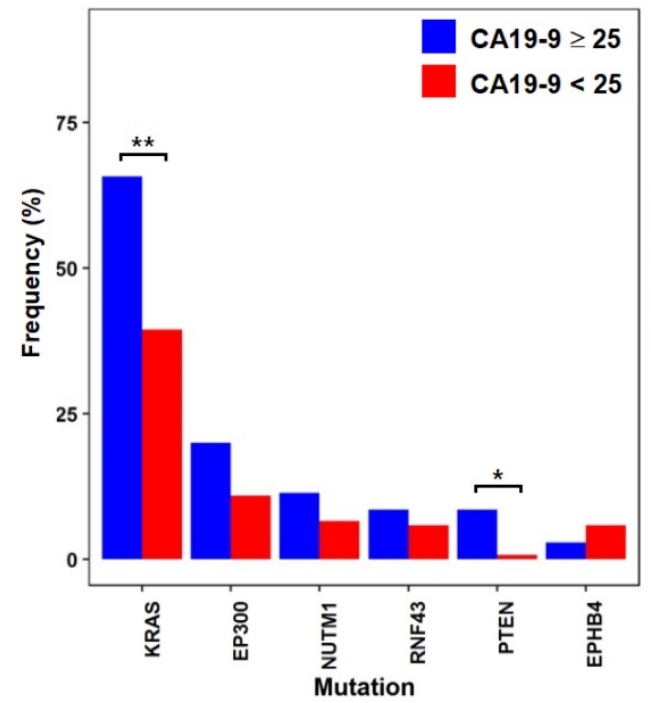

C

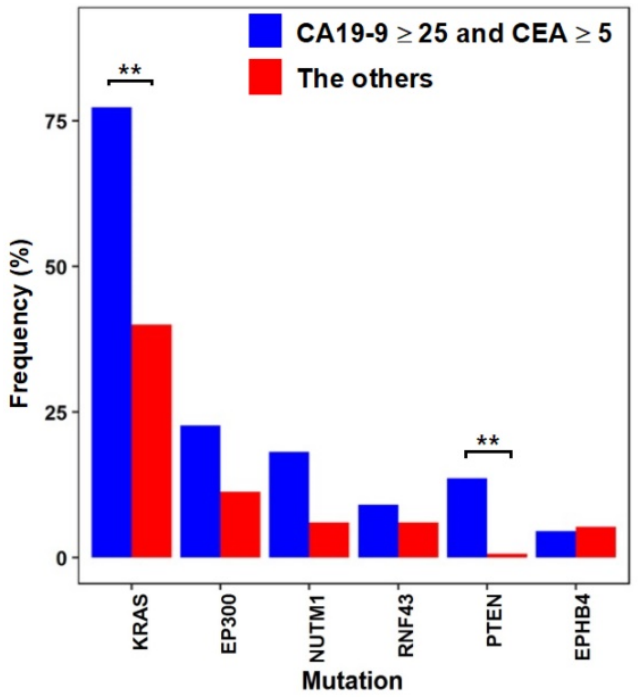

B

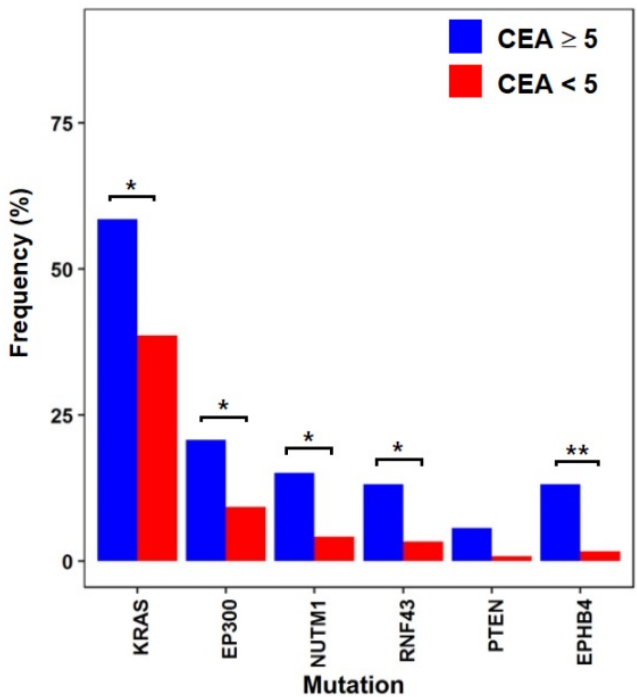

Figure 3. Differences in mutational characteristics based on cancer antigen markers from six selected genes that showed significant difference $(*: P<0.05$, ** : $<$ < 0.01) (A) Mutation frequencies from patient groups categorized by CA19-9 levels (B) Mutation frequencies from patient groups categorized by CEA levels (C) Mutation frequencies from the patient group with advanced colorectal cancer (CA19-9 $\geq 25$ and CEA $\geq 5$ ) and the other patients

\section{Mutations in association with cancer antigen markers}

CA19-9 and CEA are used as biomarkers for the diagnosis and prognosis of colorectal cancer. When comparing the mutation frequencies of individual genes from the 35 patients with CA19-9 levels greater than or equal to 25 with the mutation frequencies from the other 137 patients with CA19-9 levels lower than 25 (Fig. 3A), KRAS(CA19-9 level $\geq 25$ : 65.7\%, CA19-9 level < 25: 39.4\%; $\mathrm{p}=0.007)$ and PTEN(CA19-9 level $\geq 25$ : 8.6\%, CA19-9 level < 25: $0.7 \%, p=0.03$ ) showed significantly higher mutation frequencies. It is noteworthy that these two genes are known as common colorectal cancer mutations [15] and show correlated mutation frequencies with high CA19-9 levels. We also compared the mutation frequencies from the 53 patients with CEA levels greater than or equal to 5 with the mutation frequencies from the other 119 patients with CEA levels lower than 5 (Fig. 3B). KRAS (CEA level $\geq 5$ : $58.5 \%$, CEA level < 5: 38.7\%; p = 0.02) showed significantly high mutation frequency from the patients with the CEA level $\geq 5$, while PTEN (CEA level $\geq 5: 5.7 \%$, CEA level < 5: $0.8 \%$; $p=0.087$ ) did not show significant difference. Besides KRAS, we also observed that several cancer-associated genes show more frequent mutations from the patients with the CEA level $\geq 5$. RNF43 (CEA level $\geq 5: 13.2 \%$, CEA level $<5: 3.4 \%$; $=0.036$ ) is known as a tumor suppressor 
and negatively regulates WNT signaling [25]. EP300 (CEA level $\geq 5: 20.8 \%$, CEA level $<5: 9.2 \% ; p=0.048$ ) is a histone acetyltransferase, where its role in cell proliferation and differentiation can be associated with cancer [26]. EPHB4(CEA level $\geq 5: 13.2 \%$, CEA level < 5: $1.7 \%, p=0.004)$ mutation was also more frequent from the patients with the CEA level $\geq 5$, where previous studies have shown that it acts as a tumor suppressor [27]. By simultaneously considering both of CA19-9 and CEA levels, we evaluated the mutation frequencies from the 22 patients with high CA19-9 $(\geq 25)$ and CEA $(\geq 5)$ levels in comparison with the other 150 patients to characterize the mutations from patients with more advanced colorectal cancer. From the comparison, KRAS (CA19-9 $\geq 25$ and CEA $\geq 5: 77.3 \%$, the others: $40 \%$; $p=$ 0.001) and PTEN (CA19-9 $\geq 25$ and CEA $\geq 5: 13.7 \%$, the others: $0.7 \% ; \mathrm{p}=0.007)$ showed significantly higher mutation frequencies from the 22 patients with advanced colorectal cancer. It should be noted that the mutation frequencies of KRAS and PTEN were more significantly higher from patients with both antigen marker levels above thresholds than the patients categorized with only one antigen marker levels (see Fig. 3C in comparison with Figs. 3A and 3B).

\section{Mutational characteristics by the depth of tumor invasion}

The $\mathrm{T}$ stage of tumor represents the depth of invasion into nearby tissues, where a higher $\mathrm{T}$ stage indicates a deeper invasion of the tumor. We categorized the patients into two groups of 46 patients with low T stages (T-low; T1 and T2) and 126 patients with high $\mathrm{T}$ stages (T-high; T3 and T4), and compared the mutation frequencies of individual genes between the two groups (Fig. 4A). TP53(T-low: 56.5\%, T-high: $79.4 \% ; p=0.006$ ) and BRAF (T-low: 0\%, T-high: $11.9 \%$; $p=0.012)$, which are known frequently mutated genes in colorectal cancer, showed more frequent mutations in the patient group of $\mathrm{T} 3$ and $\mathrm{T} 4$ stages (T-high). LRP1B mutation (T-low: 17.4\%, T-high: $34.1 \%$; $\mathrm{p}=$ 0.038 ) was also more frequent at $\mathrm{T} 3$ and $\mathrm{T} 4$ stages, and this gene has been reported to inhibit the progression of colorectal cancer cells [28]. For TP53 and BRAF, their VAFs were assessed based on the $\mathrm{T}$ stages of patients' tumors (Fig. 4B). The mutation of TP53 resides across all $\mathrm{T}$ stages from $\mathrm{T} 1$ to $\mathrm{T} 4$, while it is notable that the BRAF mutations were observed in only advanced tumors of $\mathrm{T}$ stages $\mathrm{T} 3$ and $\mathrm{T} 4$. For the tumors with both of TP53 and BRAF mutations, the VAFs of these two genes are generally higher in T4 tumors than the VAFs in T3 tumors, thus implying that more advanced tumors have higher fraction of cancer cells with TP53 and BRAF mutations. We also assessed the fractions of TP53 mutation types from
T-low and T-high patient groups (Fig. 4C). The patients in the T-high group had TP53 stopgain mutations with a fraction $(14.3 \%)$ more than twice of patients from the T-low group (7.0\%), even though the difference was not statistically significant $(p=0.199)$.

\section{Genetic mutations correlated with other clinical variables}

We investigated mutations correlated with several additional clinical variables, where the mutation frequencies of individual genes were compared between patient groups that were categorized by each clinical variable (Table 2). As metastasis is one of major factors of poor prognosis for patients with colorectal cancer, mutation frequencies were compared between the group of patients without metastasis (M0, N = 144) and the other group of patients with metastasis (M1, $\mathrm{N}=28)$. BRAF mutations (M0: 6.3\%, M1: 21.4\%; $\mathrm{p}=0.019$ ) are more frequent in patients with metastasis, and this is a higher frequency compared to previously reported $5-10 \%$ in metastatic colorectal cancer [29]. EPHA6 (M0: $4.9 \%, \mathrm{M} 1: 17.9 \% ; \mathrm{p}=0.028$ ) is an ephrin receptor and has a high frequency of mutations in the group of patients with metastasis, and its association with metastasis has been previously reported for colorectal cancer [30]. We also compared the mutation frequencies the group of patients without regional lymph node metastasis (N0, N = 93) and the other group of patients with regional lymph node metastasis (N1, N = 79). The well-known APC gene for colorectal cancer [30] showed a higher mutation frequency (N0: 65.6\%, N1: 79.8\%; $\mathrm{p}=0.042)$ in patients with regional lymph node metastasis. ETV4(N0: 0\%, N1: $8.9 \% ; p=0.004$ ) also showed higher mutation frequency from patients with regional lymph node metastasis. Microsatellite instability is an important genetic marker in colorectal cancer, and it is known that patients with microsatellite instability have a better prognosis than those who do not. We compared the mutation frequencies from the microsatellite instability patient group (MSI, $\mathrm{N}=7$ ) with the frequencies from the microsatellite stability patient group (MSS, $\mathrm{N}=165$ ). TP53 (MSI: $28.6 \%$, MSS: $75.2 \%$; $\mathrm{p}=0.015$ ) and APC (MSI: 28.6\%, MSS: 73.9\%; $\mathrm{p}=$ 0.019 ) are known to be commonly mutated in colorectal cancer [15], and they showed higher mutation frequencies from the MSS group. Considering that patients with MSS generally have worse prognosis than patient of MSI, the more frequent mutations of these tumor suppressor genes from the MSS group may imply more aggressive tumorigenesis. LVI is considered as a prognostic factor and was reported to affect the survival rate of patients with colorectal cancer. From comparing the 
mutation frequencies in the 91 patients with LVI to the frequencies in the 81 patients without LVI, TP53 mutation (with LVI: 82.4\%, without LVI: 63\%; p = 0.006 ) was more frequent in the group of patients with LVI. This is another evidence that the mutation of the tumor suppressor TP53 is correlated with poor prognosis of colorectal cancer. Insulin receptor INSR (with LVI: 9.9\%, without LVI: $1.2 \%$; $\mathrm{p}=0.02$ ) also showed high mutation frequency from the patients with LVI. For correlating mutation frequencies from these colorectal cancer patients with the accompanying diagnosis status of diabetes, mutation frequencies of individual genes from 38 patients with type 2 diabetes (DM) were compared with the mutation frequencies from the other 134 patients without type 2 diabetes (non-DM). ARID1B is a chromatin remodeling factor and known to inhibit $\mathrm{WNT} / \beta$-catenin signaling [31], and it showed more frequent mutations in the diabetic group (DM: $21.1 \%$, non-DM: $7.5 \% ; \mathrm{p}=0.03$ ). TNKS2 (DM: 18.4\%, non-DM: $1.5 \% ; \mathrm{p}=0.0004)$ also showed a high frequency of mutations in the diabetic group, and it is known to be associated with the incidence of small cell lung cancer [32]. TNKS2 is known to play a role in the translocation of GLUT4, a type of glucose transporter [33], and this suggests that there can be certain impairment of glucose transport from patients with TNKS2 mutations. Regarding the status of obesity, we compared the mutation frequencies of individual genes from 45 patients with $\mathrm{BMI} \geq 25$ to the mutation frequencies from the other 127 patients with BMI < 25. RAD50 (BMI $\geq 25: 15.6 \%$, BMI < 25: 4.7\%; p $=0.042)$, a double strand break repair gene, showed a significantly higher mutation frequency in the obese patient group. This gene is known to be associated with breast cancer [34]. IRS2 (BMI $\geq 25: 8.8 \%$, BMI < 25: $1.5 \% ; p=0.041)$ is a gene involved in PI3K signaling that is related to colorectal cancer [14], and it is also known to be at the center of pathophysiology in diabetes where the aberration of IRS may be a fundamental cause of the development of insulin resistance, obesity, $\beta$ cell failure, and type 2 diabetes [35].
A

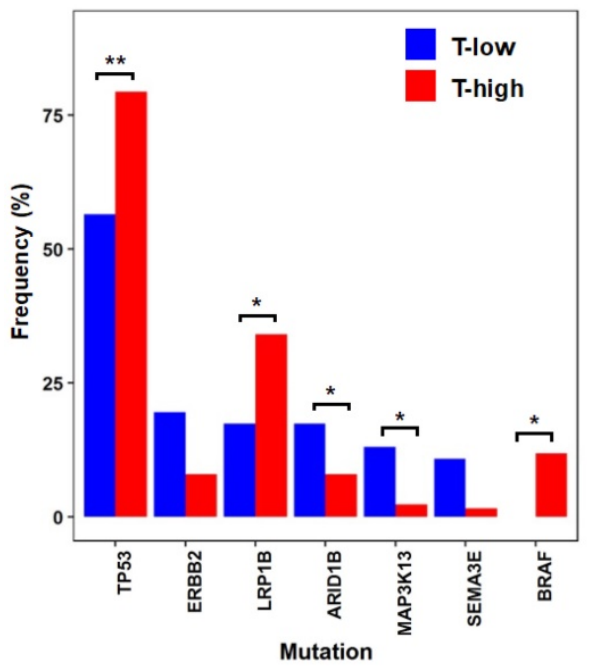

C

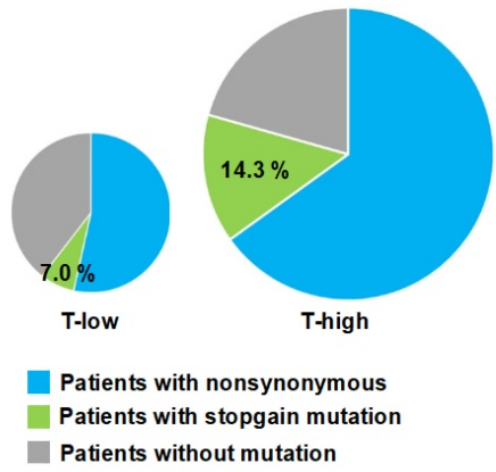

B

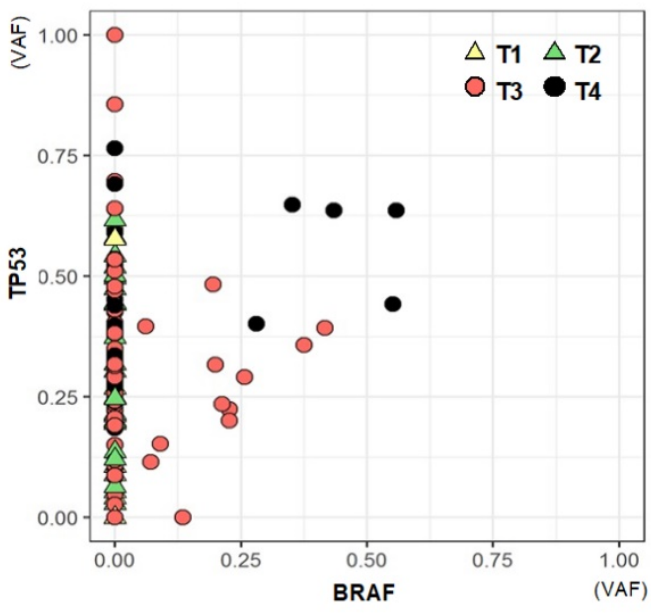

Group

Patients with nonsynonymous mutation

Patients with stopgain mutation

Patients without mutation
T-low $(\mathrm{N}=43) \quad T$-high $(\mathrm{N}=126)$

\begin{tabular}{cc}
23 & 82 \\
3 & 18 \\
17 & 26 \\
\hline
\end{tabular}

Figure 4. Mutational characteristics by tumor stages from the top seven genes that showed large differences in mutation frequencies (A) Mutation frequencies from patient groups categorized by T stages (T-low: T1 and T2, T-high: T3 and T4) $(*: p<0.05$, ** :p < 0.01) (B) Variant allele frequencies (VAFs) of TP53 and BRAF by T stages (C) The fractions of nonsynonymous and stopgain mutations of TP53 from the T-low and T-high patient groups. The size of the pie chart represents the number of patients in each group. 
Table 2. List of significant mutations that showed different frequencies between patient groups by selected clinical characteristics

\begin{tabular}{|c|c|c|c|}
\hline Genes & Categorization by clinical variable & & $\mathrm{p}$-value \\
\hline & $\begin{array}{l}\text { Without metastasis } \\
(\mathrm{N}=144)\end{array}$ & $\begin{array}{l}\text { With metastasis } \\
(\mathrm{N}=28)\end{array}$ & \\
\hline BRAF & $9,6.3 \%$ & $6,21.4 \%$ & 0.019 \\
\hline \multirow[t]{2}{*}{ EPHA6 } & $7,4.9 \%$ & $5,17.9 \%$ & 0.028 \\
\hline & $\begin{array}{l}\text { Without regional lymph node metastasis } \\
(\mathrm{N}=93)\end{array}$ & $\begin{array}{l}\text { With regional lymph node metastasis } \\
(\mathrm{N}=79)\end{array}$ & \\
\hline APC & $61,65.6 \%$ & $63,79.8 \%$ & 0.042 \\
\hline \multirow[t]{2}{*}{ ETV4 } & $0,0 \%$ & $7,8.9 \%$ & 0.004 \\
\hline & $\begin{array}{l}\text { Microsatellite stable } \\
(\mathrm{N}=165)\end{array}$ & $\begin{array}{l}\text { Microsatellite instability } \\
(\mathrm{N}=7)\end{array}$ & \\
\hline TP53 & $124,75.2 \%$ & $2,28.6 \%$ & 0.015 \\
\hline \multirow[t]{2}{*}{ APC } & $122,73.9 \%$ & $2,28.6 \%$ & 0.019 \\
\hline & $\begin{array}{l}\text { Without lymphovascular invasion } \\
(\mathrm{N}=81)\end{array}$ & $\begin{array}{l}\text { With lymphovascular invasion } \\
(\mathrm{N}=91)\end{array}$ & \\
\hline TP53 & $51,63.0 \%$ & $75,82.4 \%$ & 0.05 \\
\hline \multirow[t]{2}{*}{ INSR } & $1,1.2 \%$ & $9,9.9 \%$ & 0.02 \\
\hline & $\begin{array}{l}\text { Without diabetes mellitus } \\
(\mathrm{N}=134)\end{array}$ & $\begin{array}{l}\text { with diabetes mellitus } \\
(\mathrm{N}=38)\end{array}$ & \\
\hline ARID1B & $10,7.5 \%$ & $8,21.1 \%$ & 0.031 \\
\hline \multirow[t]{3}{*}{ TNKS2 } & $2,1.5 \%$ & $7,18.4 \%$ & 0.0004 \\
\hline & $\mathrm{BMI} \geq 25$ & $\mathrm{BMI}<25$ & \\
\hline & $(\mathrm{N}=45)$ & $(\mathrm{N}=127)$ & \\
\hline RAD50 & $7,15.6 \%$ & $6,4.7 \%$ & 0.042 \\
\hline IRS2 & $4,8.8 \%$ & $2,1.5 \%$ & 0.041 \\
\hline
\end{tabular}

Table 3. Number of genes that showed significantly different mutation frequencies by each clinical information criteria

\begin{tabular}{llll}
\hline Clinical information & $\begin{array}{l}\text { Associated mutation genes in } \\
\text { Korean }\end{array}$ & $\begin{array}{l}\text { Associated mutation genes in } \\
\text { Caucasian }\end{array}$ & $\begin{array}{l}\text { Associated mutation genes in } \\
\text { both ethnic groups }\end{array}$ \\
\hline Tumor location (Left-sided VS. Right-sided) & 22 & 59 & 5 \\
Age (ages under 50 VS. ages 50 or older) & 11 & 15 & 0 \\
BMI (BMI $\geq 25$ VS. BMI < 25) & 6 & 2 & 0 \\
TNM T stage (Low T stage(T1, T2) VS. High T stage(T3, T4)) & 9 & 30 & 0 \\
TNS N stage (Without regional lymph node metastasis VS. & 11 & 0 \\
With regional lymph node metastasis) & & 2 \\
TNM M stage (Without metastasis VS. With metastasis) & 5 & & 0
\end{tabular}

\section{Ethnic specificity of Korean mutational characteristics in comparison with the Caucasian mutational characteristics}

For each clinical information that is commonly available between our Korean subjects and the TCGA Caucasian subjects, the number of mutation genes that are significantly associated with the clinical variable is listed in Table 3 for each ethnic group along with the number of common genes between the Korean and Caucasian subjects. The complete lists of genes that showed different mutation frequencies based on clinical information criteria are given in Supplementary materials for the Korean subjects (Supplementary Table S2) and the TCGA Caucasian subjects (Supplementary Table S3).

For the mutation genes that showed significantly different mutation frequencies by tumor locations, only five genes were common between the Korean and the Caucasian. APC is a well-known gene that are often mutated in colorectal cancer, and the mutation frequency was significantly higher in left-sided tumors for both of Korean (left: $77.6 \%$, right: $57.4 \%$, p $=0.013$ ) and Caucasian (left: 84.3\%, right: 60.8\%, $\mathrm{p}=0.001)$. JAK 1 is known as a gene involved in cancer-related JAK-STAT pathway signaling, and it showed higher mutation frequencies in right-sided tumors for both of Korean (left: $1.6 \%$, right: $8.5 \%, p=$ 0.048 ) and Caucasian (left: $0 \%$, right: $10.3 \%$; $=0.005$ ). For the regional lymph node metastasis status (presence - N1, absence - N0), ACVR1B and PIK3CG showed similarly low mutation frequencies in tumors with regional lymph node metastasis for both of Korean (ACVR1B - N1: 1.3\%, N0: 9.7\%, p = 0.022; PIK3CG - N1: 0\%, N0: 7.5\%, $p=0.016)$ and Caucasian (ACVR1B - N1: 1.1\%, N0: 8.6\%, $p=0.025$; PIK3CG N1: $2.2 \%, N 0: 12.1 \%, p=0.008)$. For age, BMI, TNM T and $\mathrm{M}$ stages, there was no common mutation gene of clinical variable association between the two ethnic groups.

\section{Discussion}

Our study shows that using deep targeted sequencing for the molecular characterization of 
colorectal cancer can reveal various mutational patterns that can be correlated with clinical features. Although numerous genomic studies have been reported for colorectal cancer, few studies explored the link between mutational characteristics and various clinical features from Korean populations using next generation sequencing. From our study, TP53 and APC showed significantly higher mutation frequencies on the left-sided tumors than the right-sided tumors. A previous report [15] also showed significantly higher mutation frequency of TP53 from the left-sided tumors, but they did not report the preferential mutation of APC on the left-sided tumors with statistical significance. This discrepancy from our study in comparison with the previous report can be due to the heterogeneity in genomic characteristics from Korean populations. On the other hand, PRKDC and ATRX genes showed significantly higher mutation frequencies from the right-sided tumors. Both of these genes can be associated with more frequent observation of highly mutated MSI-high cases with right-sided tumors, as PRKDC plays a role in DNA double strand break repair [1] and ATRX is known to be associated with MSI [36]. This large molecular difference between the right and left-sided colorectal tumors may be due to different embryonic origins of left and right colon. Early onset colorectal cancer patients from our study showed more frequently mutated NOTCH1 tumor suppressor and the DNA strand break repair gene PALB2, and mutations of these genes can be related to the early tumor initiation and generally worse prognosis from young colorectal cancer patients. When the patients were categorized with the cancer antigen markers, patients with advanced colorectal cancer had more frequent KRAS and PTEN mutations. The mutation of KRAS is related with the resistance to EGFR-targeted therapeutics, and the loss of the tumor suppressor PTEN implies more aggressive tumorigenesis from these patients with advanced colorectal cancer. Another category of tumor development is the $\mathrm{T}$ stage that represents the depth of tumor invasion, and tumors with deeper invasion (T3 and $\mathrm{T} 4$ ) showed more frequent mutations of TP53 and BRAF. While the mutations of TP53 were observed across all $\mathrm{T}$ stages, only advanced tumors of $\mathrm{T} 3$ and $\mathrm{T} 4$ stages had BRAF mutations. Moreover, the VAFs of TP53 and BRAF were higher from the T4 tumors than the T3 tumors, and this suggests that advanced tumors with deeper invasion have more fraction of cancer cells with the mutations of these two genes. Regarding TP53, the ratio of patients with stopgain mutations were higher from T3 and T4 stages than from earlier T stages, and this implies the correlation between the loss of TP53 tumor suppressor and more aggressive tumor progression of colorectal cancer. In order to investigate the ethnic specificity of the identified mutational characteristics of the Korean subjects, we also analyzed the mutational characteristics of clinical association from the Caucasian subject samples of the TCGA study. The comparison of clinical variable-associated mutation genes between the two ethnic groups showed little overlap (Table 3), and only a few key colorectal cancer genes such as APC and JAK1 were common. This suggests that our results imply mutational characteristics of colorectal cancer that are specific to the Korean population in general.

We assessed the mutational characteristics of colorectal cancer from Korean patients, but there are certain limitations in our study. Firstly, we did not characterize structural variations including $\mathrm{CNVs}$, as only limited successes have been reported in identifying structural variations using targeted sequencings. Secondly, our study did not incorporate matched normal DNA and this limits the ability to detect more accurate somatic mutations from tumors. We applied stringent criteria to filter out potential germline mutations that could have been included as false-positive mutations. However, there is no method to accurately identify somatic mutations without normal DNA, and our result may include certain level of variances. Even with these limitations, we consider that our study can provide beneficial information on mutational characteristics from Korean colorectal cancer patients, and it will improve our understanding on the clinical and molecular aspects of colorectal cancer.

\section{Abbreviations}

VS.: versus; CMS: consensus molecular subtype; MSI: microsatellite instability; FFPE: formalin-fixed paraffin-embedded; IRB: institutional review board; Mb: mega bases; TML: tumor mutational load; CA: carbohydrate antigen; CEA: carcinoembryonic antigen; VAF: variant allele frequency; MSS: microsatellite stability; LVI: lymphovascular invasion; DM: type 2 diabetes mellitus; BMI: body mass index; $\mathrm{CNV}$ : copy number variation.

\section{Supplementary Material}

Supplementary tables. https://www.jcancer.org/v12p7300s1.pdf

\section{Acknowledgements}

This work was supported by the National Research Foundation of Korea (NRF) grant funded by the Korea government (MSIT) (No. NRF-2021R1A5A2030333) to WL. This work was also 
supported by the Gachon University research fund of 2020 (GCU-202002550001), funded to SJ.

\section{Competing Interests}

The authors have declared that no competing interest exists.

\section{References}

1. Weiss JM, Schumacher J, Allen GO, Neuman H, Lange EO, Loconte NK, et al. Adjuvant chemotherapy for stage II right-sided and left-sided colon cancer: analysis of SEER-medicare data. Ann Surg Oncol. 2014; 21: 1781-91.

2. Guinney J, Dienstmann R, Wang X, de Reynies A, Schlicker A, Soneson C, et al. The consensus molecular subtypes of colorectal cancer. Nat Med. 2015; 21: 1350-6.

3. Petrelli F, Tomasello G, Borgonovo K, Ghidini M, Turati L, Dallera P, et al. Prognostic Survival Associated With Left-Sided vs Right-Sided Colon Cancer: A Systematic Review and Meta-analysis. JAMA Oncol. 2017; 3: 211-9.

4. Lee MS, Menter DG, Kopetz S. Right Versus Left Colon Cancer Biology: Integrating the Consensus Molecular Subtypes. J Natl Compr Canc Netw. 2017; 15: 411-9.

5. Lieu CH, Golemis EA, Serebriiskii IG, Newberg J, Hemmerich A, Connelly C, et al. Comprehensive Genomic Landscapes in Early and Later Onset Colorectal Cancer. Clin Cancer Res. 2019; 25: 5852-8.

6. Shen Y, Han X, Wang J, Wang S, Yang H, Lu SH, et al. Prognostic impact of mutation profiling in patients with stage II and III colon cancer. Sci Rep. 2016; 6: 24310.

7. Poulos RC, Perera D, Packham D, Shah A, Janitz C, Pimanda JE, et al. Scarcity of Recurrent Regulatory Driver Mutations in Colorectal Cancer Revealed by Targeted Deep Sequencing. JNCI Cancer Spectr. 2019; 3: pkz012.

8. Bordonaro M, Lazarova D. Hypothesis: Obesity Is Associated with a Lower Mutation Threshold in Colon Cancer. J Cancer. 2015; 6: 825-31.

9. Byun JH, Ahn JB, Kim SY, Kang JH, Zang DY, Kang SY, et al. The impact of primary tumor location in patients with metastatic colorectal cancer: a Korean Cancer Study Group CO12-04 study. Korean J Intern Med. 2019; 34: 165-77.

10. Boland CR, Thibodeau SN, Hamilton SR, Sidransky D, Eshleman JR, Burt RW, et al. A National Cancer Institute Workshop on Microsatellite Instability for cancer detection and familial predisposition: development of international criteria for the determination of microsatellite instability in colorectal cancer. Cancer Res. 1998; 58: 5248-57.

11. Kim S, Park C, Ji Y, Kim DG, Bae H, van Vrancken M, et al. Deamination Effects in Formalin-Fixed, Paraffin-Embedded Tissue Samples in the Era of Precision Medicine. J Mol Diagn. 2017; 19: 137-46.

12. Bhagwate AV, Liu Y, Winham SJ, McDonough SJ, Stallings-Mann ML, Heinzen EP, et al. Bioinformatics and DNA-extraction strategies to reliably detect genetic variants from FFPE breast tissue samples. BMC Genomics. 2019; 20: 689 .

13. Trevino V. HotSpotAnnotations-a database for hotspot mutations and annotations in cancer. Database (Oxford). 2020; 2020.

14. Cancer Genome Atlas N. Comprehensive molecular characterization of human colon and rectal cancer. Nature. 2012; 487: 330-7.

15. Salem ME, Weinberg BA, Xiu J, El-Deiry WS, Hwang JJ, Gatalica Z, et al. Comparative molecular analyses of left-sided colon, right-sided colon, and rectal cancers. Oncotarget. 2017; 8: 86356-68.

16. Wechsler T, Chen BP, Harper R, Morotomi-Yano K, Huang BC, Meek K, et al. DNA-PKcs function regulated specifically by protein phosphatase 5 . Proc Natl Acad Sci U S A. 2004; 101: 1247-52.

17. Koschmann C, Calinescu AA, Nunez FJ, Mackay A, Fazal-Salom J, Thomas D, et al. ATRX loss promotes tumor growth and impairs nonhomologous end joining DNA repair in glioma. Sci Transl Med. 2016; 8: 328ra28.

18. Kudithipudi S, Lungu C, Rathert P, Happel N, Jeltsch A. Substrate specificity analysis and novel substrates of the protein lysine methyltransferase NSD1. Chem Biol. 2014; 21: 226-37.

19. Classon M, Harlow E. The retinoblastoma tumour suppressor in development and cancer. Nat Rev Cancer. 2002; 2: 910-7.

20. Kreiling JL, Montgomery MA, Wheeler JR, Kopanic JL, Connelly CM, Zavorka $\mathrm{ME}$ et al. Dominant-negative effect of truncated mannose 6-phosphate/insulin-like growth factor II receptor species in cancer. FEBS J. 2012; 279: 2695-713.

21. Nowell CS, Radtke F. Notch as a tumour suppressor. Nat Rev Cancer. 2017; 17: 145-59.

22. Wu X, Bekker-Jensen IH, Christensen J, Rasmussen KD, Sidoli S, Qi Y, et al. Tumor suppressor ASXL1 is essential for the activation of INK4B expression in response to oncogene activity and anti-proliferative signals. Cell Res. 2015; 25: 1205-18.

23. Evans MK, Longo DL. PALB2 mutations and breast-cancer risk. N Engl J Med. 2014; 371: 566-8

24. Smogorzewska A, Matsuoka S, Vinciguerra P, McDonald ER, 3rd, Hurov KE, Luo J, et al. Identification of the FANCI protein, a monoubiquitinated FANCD2 paralog required for DNA repair. Cell. 2007; 129: 289-301.
25. Koo BK, Spit M, Jordens I, Low TY, Stange DE, van de Wetering M, et al. Tumour suppressor RNF43 is a stem-cell E3 ligase that induces endocytosis of Wnt receptors. Nature. 2012; 488: 665-9.

26. Gayther SA, Batley SJ, Linger L, Bannister A, Thorpe K, Chin SF, et al. Mutations truncating the EP300 acetylase in human cancers. Nat Genet. 2000; 24: $300-3$.

27. Dopeso H, Mateo-Lozano S, Mazzolini R, Rodrigues P, Lagares-Tena L, Ceron $\mathrm{J}$, et al. The receptor tyrosine kinase EPHB4 has tumor suppressor activities in intestinal tumorigenesis. Cancer Res. 2009; 69: 7430-8.

28. Wang Z, Sun P, Gao C, Chen J, Li J, Chen Z, et al. Down-regulation of LRP1B in colon cancer promoted the growth and migration of cancer cells. Exp Cell Res. 2017; 357: 1-8.

29. Korphaisarn K, Kopetz S. BRAF-Directed Therapy in Metastatic Colorectal Cancer. Cancer J. 2016; 22: 175-8.

30. Mathot L, Kundu S, Ljungstrom V, Svedlund J, Moens L, Adlerteg T, et al. Somatic Ephrin Receptor Mutations Are Associated with Metastasis in Primary Colorectal Cancer. Cancer Res. 2017; 77: 1730-40.

31. Vasileiou G, Ekici AB, Uebe S, Zweier C, Hoyer J, Engels $H$, et al. Chromatin-Remodeling-Factor ARID1B Represses Wnt/beta-Catenin Signaling. Am J Hum Genet. 2015; 97: 445-56.

32. Wang Y, Jiang W, Liu X, Zhang Y. Tankyrase 2 (TNKS2) polymorphism associated with risk in developing non-small cell lung cancer in a Chinese population. Pathol Res Pract. 2015; 211: 766-71.

33. Guo HL, Zhang C, Liu Q, Li Q, Lian G, Wu D, et al. The Axin/TNKS complex interacts with KIF3A and is required for insulin-stimulated GLUT4 translocation. Cell Res. 2012; 22: 1246-57.

34. Heikkinen K, Rapakko K, Karppinen SM, Erkko H, Knuutila S, Lundan T, et al. RAD50 and NBS1 are breast cancer susceptibility genes associated with genomic instability. Carcinogenesis. 2006; 27: 1593-9.

35. Brady MJ. IRS2 takes center stage in the development of type 2 diabetes. J Clin Invest. 2004; 114: 886-8.

36. Malas MA, Aslankoc R, Ungor B, Sulak O, Candir O. The development of large intestine during the fetal period. Early Hum Dev. 2004; 78: 1-13. 